\title{
Una propuesta de evaluación en el EEES: el uso del portfolio en una clase de idiomas
}

\author{
María Martínez LiRola \\ Departamento de Filología Inglesa. Universidad de Alicante
}

Recibido: 18 Abril 2007 / Aceptado: 22 Junio 2007

ISSN: $1697-7467$

\begin{abstract}
This article is based on putting into practice methodological experiences based on the philosophy of the European Higher Education such as collaborative learning and autonomous learning.

Apart from this, the article is also based on using new criteria and procedures to evaluate students in such a way that the final exam is not the only grade to base students' learning process. Our end was to emphasize that students are the protagonists of the teachinglearning process and not just passive receivers of the information selected by the lecturer. To put into practice these ideas related to the European Credit Transfer System, we decided to evaluate students using a portfolio in a core subject of the third year of English Studies. The results show that students learn more and get better results.

Key words: European Framework, evaluation, portfolio, autonomous learning.
\end{abstract}

RESUMEN: Este artículo es el resultado de la puesta en práctica de experiencias metodológicas acordes con el Espacio Europeo de Educación Superior como el aprendizaje colaborativo y el aprendizaje autónomo así como del diseño y de la puesta en práctica de nuevos criterios y procedimientos de evaluación de modo que el examen final no tenga todo el peso de la nota de la asignatura. Nuestro fin era potenciar la idea de que el alumno es el protagonista del proceso de enseñanza-aprendizaje y no un mero receptor pasivo de las informaciones seleccionadas por el profesor. Para llevar a cabo estas ideas acordes con la filosofía de la convergencia europea y el EEES, decidimos implantar una evaluación basada en el uso del portfolio en una asignatura troncal de tercero de Filología Inglesa. Los resultados ponen de manifiesto que los alumnos aprenden más y obtienen mejores resultados.

Palabras clave: EEES, evaluación, portfolio, aprendizaje autónomo.

\section{INTRODUCCIÓN}

Nos encontramos en un momento de transición en la educación superior en el que la universidad ha de hacer un esfuerzo por adaptar sus funciones de creación y transmisión del conocimiento a la sociedad. Esto implica una reforma profunda en la metodología y en la organización de la enseñanza superior de modo que las enseñanzas universitarias den respuesta a las demandas de la sociedad y contribuyan a formar ciudadanos activos, con capacidad 
crítica y comprometidos con su entorno. Esta reforma ha de estar basada "en la flexibilidad, en la transversalidad y en la multidisciplinariedad como mecanismo de respuesta a las necesidades de una nueva sociedad." (MEC, 2006: 27)

La publicación del Common European Framework of Reference for Languages: Learning, Teaching, Assessment (Council of Europe, 2001) se ha convertido en un punto de referencia indispensable para la convergencia europea en cuestiones relacionadas con la educación:

The Common European Framework provides a common basis for the elaboration of language syllabuses, curriculum guidelines, textbooks, etc. across Europe. It describes in a comprehensive way what language learners have to learn to do in order to use a language for communication and what knowledge and skills they have to develop so as to be able to act effectively. The description also covers the cultural context in which language is set. The Framework also defines levels of proficiency which allows learners' progress to be measured at each stage of learning on a lifelong basis. (Council of Europe, Common European Framework, 2001:1).

Es bien sabido que el proceso de Convergencia Europea que debe verse completado en el año 2010, tiene cinco pilares fundamentales: I. Créditos europeos: ECTS, II. La enseñanza por competencias, III. Aprendizaje autónomo, IV. Aprendizaje a lo largo de la Vida (Lifelong Learning) y V. Materiales didácticos. En este artículo nos proponemos centrarnos en uno de esos cinco pilares: el aprendizaje autónomo y a su relación con las nuevas formas de evaluación.

El aprendizaje autónomo es una forma de aprendizaje en la que el estudiante asume una parte importante de la responsabilidad de la organización de su trabajo ajustándola a su propio ritmo, en palabras de Rico Vercher y Rico Pérez (2004:15): “El aprendizaje autónomo, fundamento del acontecer discente, es una modalidad de aprendizaje en la que el alumno se responsabiliza de la organización de su trabajo, de la adquisición de conocimientos y los asimila a su propio ritmo."

Las características principales de este tipo de aprendizaje son las que enumeramos a continuación:

Para ello han de darse una serie de condiciones que enumeramos a continuación:

- Requiere de un sistema intenso de tutoría.

- Exige mayor esfuerzo docente que las lecciones.

- Exige una preparación del alumnado en el dominio de ciertas técnicas de trabajo (incluidas las TIC).

- Requiere poseer capacidades y habilidades genéricas y transferibles a cualquier situación de aprendizaje (manejo de fuentes, gestión información, etc.).

- Requiere de una nueva actitud de profesores y alumnos (sobre todo de éstos).

- Requiere infraestructuras tecnológicas y didácticas. Con este aprendizaje conseguimos que el alumno sea autónomo, es decir, un alumno con las siguientes competencias:

- Requiere capacidad de iniciativa.

- Saber configurar un plan de trabajo realista.

- Manejarse con fuentes de información (y saber contrastarlas).

- Comprender informaciones y textos. Resumirlos. 
- Plantear y resolver problemas.

- Voluntad por conocer cosas nuevas y profundizar en ellas.

- Transferir, extrapolar y aplicar conocimientos a situaciones nuevas.

- Reflexionar y evaluar sobre su propio trabajo

Una de las ideas fundamentales del EEES consiste en desarrollar la autonomía del alumno y en ayudar a los alumnos a tomar conciencia de las diferentes estrategias que pueden potenciar su aprendizaje teniendo en cuenta sus características individuales. Concurrimos con Martínez Lirola (2007:41) en que:

Potenciar que el aprendizaje esté centrado en el alumno implica un cambio en el papel del profesor y una adecuación de las metodologías docentes al aprendizaje presencial y no presencial en la que la tutoría juega un papel fundamental, así como una organización de la docencia en la que se presta especial atención al tiempo y al esfuerzo que el alumno emplea, de modo que se fomente el aprendizaje autónomo.

El alumno se ha de convertir en un ciudadano activo que participe en la vida social. De ahí que la educación superior ha de crear "ambientes de aprendizaje en los que cada estudiante pueda desarrollar tanto capacidades críticas como capacidades de participación en la creación de la cultura." (Martínez Ruiz y Sauleda Parés, 2005:13).

Como docentes, hemos de tener en cuenta en todo momento que el aprendizaje es un proceso global en el que intervienen diferentes factores: el contexto, las actitudes y expectativas de los alumnos, la formación del docente, etc. Por esta razón, hemos de tener presente que el profesorado debe estar formado en didáctica, independientemente de cual sea su especialidad, en palabras de Colen et al. (2006:3-14):

El profesorado no debe sólo actualizarse en su área de conocimiento, sino que también debe formarse como docente, debe ser competente en la producción y el diseño de recursos didácticos (aplicando las tecnologías de la información y comunicación que se vayan generando en cada momento), en la gestión del aula universitaria, en la creación, el seguimiento y la evaluación de situaciones ricas, motivadoras y generadoras de aprendizaje parra los alumnos.

Este artículo es el resultado del diseño y de la puesta en práctica de nuevos criterios y procedimientos de evaluación de modo que el examen final no tenga todo el peso de la nota de la asignatura (vid. apartado 2). Nuestro fin es potenciar la idea de que el alumno es el protagonista del proceso de enseñanza-aprendizaje y no un mero receptor pasivo de las informaciones seleccionadas por el profesor, en palabras de Martínez Ruiz y Sauleda Parés (2005: 14): "Diseñar un aprendizaje centrado en el alumno, requiere una perspectiva integral en la consideración del alumnado y sus competencias cognitivas, capacidades metacognitivas y disposiciones personales y sociales." 


\section{La evaluación en EL EeEs: El PORTFOlio COMO ELEMENTO FUNDAMEN- TAL EN LA EVALUACIÓN}

Un punto muy importante en la organización y planificación de la clase es la forma de evaluación pues afecta a todo el proceso de enseñanza-aprendizaje, en palabras de PérezParedes y Rubio (2005:606-607): "Evaluation considers the teaching and learning program as a whole, and seeks to obtain feedback that can serve different purposes for the different agents in education, from teachers to curriculum designers."

La evaluación debe, por consiguiente, estar en consonancia con los objetivos y las competencias globales especificados en los programas de las asignaturas. Por esta razón es importante distribuir concienzudamente las ponderaciones de cada actividad que forme parte de la evaluación y que el alumno las conozca. Los criterios para calificar deben ser decididos por cada profesor con base en su estilo personal y plan de trabajo. Pensamos que debe dársele importancia a la evaluación de las prácticas frente a la tradicional del examen puesto que en aquellas se hace un mejor seguimiento del trabajo desempeñado por el alumno a lo largo del curso. En general podemos hablar de cuatro tipos de evaluación: diagnóstica, inicial, formativa o continua y sumativa o final.

La evaluación diagnóstica se emplea para saber si el alumno es capaz de aprender una asignatura, es decir, para observar si los conocimientos y la capacidad intelectual del alumno son los adecuados para alcanzar las competencias que se pretenden alcanzar en la asignatura. Este tipo de evaluación no está restringida a ningún tipo de asignatura.

La evaluación inicial se utiliza para saber cuáles son los conocimientos previos de los alumnos. Normalmente se lleva a cabo al comenzar el curso académico de modo que el profesor pueda implementar las modificaciones que considere necesarias en el programa de la asignatura. Con este tipo de evaluación el profesor también puede observar las diferencias individuales y adaptar el currículum a determinados alumnos.

La evaluación formativa o continua proporciona al profesor información por medio del trabajo del alumno, su actitud y su comportamiento. En este tipo de evaluación se tienen en cuenta los deberes, las composiciones, ejercicios escritos, la participación en clase, etc. Según Pérez-Paredes y Rubio (2005:610), este tipo de evaluación tiene un doble propósito: "first, it helps teachers control the process of learning; second it should inform and motivate students." Normalmente a este tipo de evaluación se le critica que suele acabar con la motivación del alumno porque el aprendizaje se convierte en un fin instrumental.

La evaluación sumativa tiene lugar al final del proceso de aprendizaje por medio de las clases, los deberes y el estudio. También se denomina evaluación final si se evalúa todo el curso. El instrumento empleado en este tipo de evaluación para recopilar información sobre el aprendizaje de los alumnos son exámenes escritos u orales.

A continuación incluimos una propuesta de evaluación que se llevó a cabo en el curso académico 2005-2006 en la que los exámenes tienen un 50\% de la calificación total y el resto de la nota se obtiene de la siguiente manera: el $30 \%$ de la nota consistirá en la evaluación de un portfolio en el que el alumno vaya acumulando materiales y el $20 \%$ restante en la asistencia y participación del alumno a clase: 
Tabla 1. Procedimientos de evaluación en consonancia con el EEES

\begin{tabular}{|c|c|c|c|}
\hline Aspecto & Criterios & Instrumento & Peso \\
\hline Conceptos de la materia & $\begin{array}{l}\text { - Dominio de los } \\
\text { conocimientos teóricos } \\
\text { de la materia. } \\
\text { - Claridad en la descripción } \\
\text { de los conceptos teóricos } \\
\text { exigidos. } \\
\text { - Aplicación de los } \\
\text { conceptos teóricos a } \\
\text { textos escritos en inglés. }\end{array}$ & $\begin{array}{l}\text { Se realizará al final del } \\
\text { cuatrimestre unas prueba } \\
\text { escrita, puntuándose entre } 0 \\
\text { y } 5 \text { (Prueba objetiva) }\end{array}$ & $\begin{array}{l}50 \%(25 \% \text { el examen de } \\
\text { cada cuatrimestre })\end{array}$ \\
\hline Portfolio & $\begin{array}{l}\text { En cada portfolio se } \\
\text { analizará: } \\
\text { - Originalidad } \\
\text { - Ortografía y presentación } \\
\text { - Bibliografía empleada } \\
\text { - Calidad de la } \\
\text { documentación } \\
\text { - Estructura en la } \\
\text { exposición y valoración } \\
\text { de las conclusiones } \\
\text { alcanzadas } \\
\text { - valoración crítica de las } \\
\text { ideas y argumentos de } \\
\text { los autores trabajados } \\
\end{array}$ & $\begin{array}{l}\text { - Entrega de los materiales } \\
\text { requeridos por el } \\
\text { profesor y de ejercicios } \\
\text { prácticos } \\
\text { - Se entregará al final del } \\
\text { primer cuatrimestre y al } \\
\text { final del segundo }\end{array}$ & $\begin{array}{l}30 \% \text { (15\% cada uno del los } \\
\text { materiales del primer y } \\
\text { segundo cuatrimestre) }\end{array}$ \\
\hline Asistencia y participación & $\begin{array}{l}\text { - Participación activa en } \\
\text { clase } \\
\text { - Participación de los } \\
\text { debates organizados a } \\
\text { través del campus virtual } \\
\text { - Participación en las } \\
\text { prácticas grupales de } \\
\text { clase } \\
\text { - Realización de los } \\
\text { ejercicios propuestos en } \\
\text { clase y en el campus } \\
\text { virtual }\end{array}$ & $\begin{array}{l}\text { - Observación y notas del } \\
\text { profesor }\end{array}$ & $20 \%$ \\
\hline
\end{tabular}

En el cuadro anterior se puede observar que se concede un papel importante en la evaluación al uso del portfolio, también denominado carpeta de aprendizaje. Éste consiste en una carpeta de trabajo en la que el alumno recopila distintos trabajos que han sido diseñados para adquirir las competencias que como objetivo se marca el docente en su disciplina, y puede servir para que los alumnos mejoren su nivel de escritura, en palabras de Celce Murcia y Olshtain (2000:159):

The portfolio, which is usually an ongoing collection of different writing assignments kept by the student in a folder or workbook, has become an important concept in developing writing skills and in giving teachers a fairer and more perceptive way to evaluate. 
El portfolio es un documento muy útil en la evaluación para mostrar el progreso de los alumnos así como sus logros. Siguiendo a Brown (2001:418), el portfolio puede incluir ensayos, composiciones, poemas, reseñas de libros, grabaciones de video o de cintas, y cualquier otra actividad que el profesor decida especificar. El uso del portfolio está muy relacionado con el aprendizaje basado en proyectos (project-based learning). Se trata de pequeños trabajos de investigación que formarían parte del portfolio de los alumnos y constituirían otro índice fiable de evaluación.

El portfolio es una forma auténtica de evaluación pues permite establecer una vinculación entre la teoría y la práctica, así como una buena herramienta para la reflexión. Además, el portfolio es un modo de evaluación muy fiable siempre y cuando se haya organizado de modo que:

- Ayude a los estudiantes a asumir responsabilidades sobre el propio aprendizaje, al implicarse en el proceso de evaluación.

- Proporcione información detallada al profesorado.

- Integre la evaluación en el proceso de aprendizaje.

- Estimule tanto a docentes como a estudiantes a introducir cambios en las formas de enseñanza y aprendizaje.

- Permite organizar y dar coherencia a la información tratada. (Colen et al., 2006 : 108)

Tal y como apuntan Colen et al. (2006:84), pensamos que el portfolio mejora el modo de aprendizaje de los alumnos y compartimos las siguientes relaciones entre evaluación y carpeta de aprendizaje:

1. Evaluación continuada, procesual o durante la secuencia didáctica.

2. Evaluación compleja y multidimensional.

3. Evaluación orientada a la retroacción o evaluación pedagógica.

4. Evaluación contextualizada o situacional y personalizada o singularizada.

5. Evaluación colaborativa.

\section{UNA EXPERIENCIA PRÁCTICA DEL USO DEL PORTFOLIO EN LA ENSEÑANZA UNIVERSITARIA}

Con esta experiencia nos propusimos llevar a la práctica nuevos modos de evaluación en los que el examen fuera una nota más y no el único elemento en la evaluación. Para evaluar los conocimientos se concedió importancia a otras herramientas como el uso del portfolio y la asistencia y participación del alumno en clase (vid. tabla 1). Con el portfolio pretendíamos que los alumnos guardaran en esta carpeta de trabajo todos los borradores y diferentes versiones de las tareas que fueran requeridas por la profesora. Periódicamente, la profesora citó a los alumnos a una tutoría individualizada para dialogar sobre el desarrollo de las actividades escritas y la elaboración del portfolio.

Siguiendo a Escobar (2001:349), nos propusimos seguir los siguientes pasos para ayudar a los alumnos a crear y mantener el portfolio, de modo que se pudiera observar su utilidad en el proceso de enseñanza-aprendizaje:

- Elegir un número de tareas relacionadas con los objetivos y las competencias de aprendizaje. 
- Definir los criterios de evaluación con claridad.

- Diseñar una plantilla de autoevaluación de modo que los alumnos puedan realizar la tarea y después evaluar el resultado.

- Cuando el profesor cite a los alumnos a una tutoría individualizada, éstos han de seleccionar las mejores tareas y escribir un pequeño informe explicando por qué han seleccionado esas tareas.

- El profesor evalúa las tareas de los alumnos de acuerdo con los criterios establecidos con anterioridad y conocidos tantos por los alumnos como por los profesores.

- El profesor y el alumno comentan el portfolio en una tutoría individualizada.

Se ofreció este modo de evaluación a todos los alumnos del grupo B matriculados en la asignatura Gramática Inglesa III en el segundo cuatrimestre. Eran un total de 68 alumnos, de los cuales 30 decidieron ser evaluados siguiendo los criterios anteriormente presentados. El resto fueron evaluados de manera tradicional teniendo una sola nota obtenida mediante su examen final al acabar el cuatrimestre. La única razón por la que esta experiencia no se desarrolló durante todo el curso fue porque en el primer cuatrimestre la profesora tenía un total de 246 alumnos en diferentes asignaturas; debido a la carga de trabajo añadido que implica la corrección y la tutorización del portfolio, a la profesora le pareció pertinente llevar a cabo esta experiencia piloto de evaluación siguiendo los criterios propuestos por el EEES cuando tuviera menos alumnos. Con el portfolio que se pidió a los alumnos matriculados en la asignatura troncal Gramática Inglesa III de tercero de Filología Inglesa pretendíamos que los alumnos alcanzasen los siguientes objetivos:

- Hacer uso de recursos para la investigación.

- Animarte a que revises tu escritura antes de entregarla al profesor.

- Analizar los recursos para estructurar un texto.

- Aprender a escribir un trabajo académico.

- Sintetizar información.

- Llevar a cabo una presentación oral.

- Participar activamente en un debate.

Además de los objetivos que acabamos de enumerar, es fundamental poner de manifiesto que evaluar a los alumnos con el portfolio tiene como finalidad que el alumno aprenda de manera autónoma, es decir, que sea un alumno que haya desarrollado las siguientes competencias:

- Capacidad de iniciativa.

- Saber configurar un plan de trabajo realista.

- Manejarse con fuentes de información (y saber contrastarlas).

- Comprender informaciones y textos. Resumirlos.

- Plantear y resolver problemas.

- Voluntad por conocer cosas nuevas y profundizar en ellas.

- Transferir, extrapolar y aplicar conocimientos a situaciones nuevas.

- Reflexionar y evaluar sobre su propio trabajo. (Martínez Lirola, 2007: 37)

A nuestro juicio, el portfolio facilita el aprendizaje autónomo de los alumnos, pues les ofrece la posibilidad de seleccionar las fuentes de información que ellos estimen oportunas 
para llevar a cabo las tareas y hacen que se responsabilicen de su aprendizaje al llevar a cabo de manera autónoma cada una de las tareas. Esto va unido a los nuevos papeles que el profesor ha de asumir en el EEES, tal y como señala Martínez Lirola (2007:34):

Con la creación del Espacio Europeo de Educación Superior (EEES), se produce un cambio en el papel del profesor pues de ser el protagonista de la enseñanza al ser la persona que estructura el proceso de aprendizaje, el supervisor y director de trabajos, pasa a ser "un acompañante en el proceso de aprender, que ayuda al que estudia a alcanzar ciertas competencias” (González y Wagenaar, 2003:74).

El aprendizaje autónomo también se fomenta claramente con este modo de evaluación porque con el portfolio el profesor cede poder a los alumnos y pierde protagonismo pues las clases magistrales caracterizadas por la transmisión unilateral de conocimientos se ven reemplazadas por unas clases en las que los protagonistas son los estudiantes en las que predomina la interacción, es decir, en el nuevo paradigma educativo propuesto por el EEES, observamos una serie de cambios, entre los que destacamos los siguientes:

[...] el profesor deja de ser el centro, el punto de referencia en el planteamiento educativo y se convierte en tutor del alumno, lo que le exige tareas que pueden resultar en buena medida novedosas: una programación previa y una planificación al detalle de todas las actividades que va a llevar a cabo; una interacción necesaria con el alumno; una coordinación con compañeros de departamento y/o de materia específica en favor de la coherencia y la idoneidad del programa de estudios; un "sacrificio" de contenidos específicos en favor de la potenciación de competencias procedimentales que garanticen la autonomía intelectual del alumno, etc. (Martínez Lirola, Peñalver, Ponce, Puche y Santacreu, 2007:2).

En el apartado 2 de este artículo pusimos de manifiesto que el portfolio es una herramienta muy útil en la evaluación pero supone mucha más carga de trabajo en términos de tiempo y esfuerzo tanto para profesores como para alumnos. Los alumnos ven compensado su esfuerzo porque obtienen mejores calificaciones y suelen coincidir en que aprenden más (vid. apartado 4); el profesorado, por el contrario, no ve recompensado de ningún modo el trabajo extra que hace por lo que el tiempo que supone el diseño de un portfolio coherente con la programación del curso y su utilización en el aula es una de las principales inquietudes del profesorado universitario:

De igual forma, para el profesorado el trabajo con la carpeta constituye una importante carga en términos de tiempo y esfuerzo pues es mucho el tiempo necesario para las tutorías, la negociación, el consenso, la explicación, la orientación de los equipos de trabajo, las revisiones (interactivas o no) frecuentes y la devolución de los trabajos, etc. (Colen et al., $2006: 119$ ).

\subsection{Catálogo de productos del portfolio}

El catálogo de productos que configuran el portfolio que proponemos ha sido seleccionado teniendo en cuenta las siguientes características que según Vez (2001:270) ha de tener toda actividad efectiva en el proceso de aprendizaje: 
- Representativa de procesos de comunicación en la vida real.

- Identificable como unidad de actividad en el aula.

- Dirigida intencionalmente hacia el aprendizaje de la lengua.

- Diseñada con un objetivo, estructura y secuencia del trabajo.

- Orientada a la consecución de un objetivo de manipulación de información y significados.

A continuación, ofrecemos, a modo orientativo, una propuesta de actividades a incluir en el portfolio para cada uno de los temas que componen el segundo cuatrimestre de la asignatura tomada como muestra en este trabajo, que corresponde a los procesos de formación de palabras en inglés:

Tarea del tema 7 (10\%), "Compounding": una vez estudiadas las referencias bibliográficas presentadas en clase, el alumno ha de preparar un esquema que sea una clasificación exhaustiva de los compuestos en inglés en la que se recojan todos los aspectos presentados por los autores estudiados. Además, ha de escribir un pequeño ensayo en el que justifique por qué lo ha hecho así y las principales características de las clasificaciones de cada autor. Se valorará la consulta referencias de la bibliografía suplementaria. Con esta actividad se pretende que los alumnos lean las principales referencias bibliográficas relacionadas con los compuestos en inglés, de modo que estén familiarizados con los principales autores que escriben sobre este tema. Además, se pretende que tengan capacidad de síntesis y que sean capaces de organizar sus ideas con un esquema personal. Finalmente, con el ensayo se pretende fomentar la capacidad crítica del alumno.

Tarea del tema 8 (5\%), "Derivation": por grupos que se establecerán en clase de entre 3 y 5 personas cada uno, se trabajarán las principales características de algunos prefijos y sufijos en inglés con el fin de presentar en clase una presentación oral de 5 a 10 minutos por cada grupo. Los alumnos han de preparar un esquema que se le entregará a la profesora antes de la presentación oral. En la presentación se prestará atención al uso fluido del inglés. En esta ocasión la actividad se ha organizado con el fin de que los alumnos trabajen por grupos y tengan que tomar decisiones en equipo, negociar el modo en que preparan el esquema que han de entregar a la profesora y consensuar la manera en que preparan su presentación. Esta actividad del portfolio también tiene un aspecto didáctico pues los alumnos han de explicar a la profesora y a sus compañeros el esquema de un modo claro y pedagógico, aspectos que serán tenidos en cuenta en su evaluación. Todos los esquemas presentados por los alumnos serán colgados en el campus virtual por la profesora de modo que todos los alumnos se puedan beneficiar del trabajo realizado por sus compañeros.

Tarea del tema 9 (3\%), "Conversion": los alumnos trabajarán por grupos sobre una batería de ejercicios prácticos relacionados con la conversión en inglés que serán corregidos en clase. En clase se resolverán todas las dudas y se contemplarán todas las respuestas posibles de modo que se fomente la interacción en el aula. Algunos de los ejercicios serán proporcionados por la profesora de modo que sirvan de modelo pero otros han de ser buscados por los alumnos, de modo que pasen tiempo en la biblioteca seleccionando ejercicios prácticos que les sirvan para poner en práctica los principales aspectos teóricos explicados por la profesora. En el campus virtual se colgará una lista de libros en la que los alumnos puedan encontrar ejercicios de modo que la búsqueda de las actividades prácticas sea guiada. Una vez que los alumnos hayan seleccionado los ejercicios, tendrán una tutorías obligatoria con la profesora para comentar los criterios que han seguido para seleccionar los ejercicios. 
Tarea del tema 10 (7\%), "Shortenings": por grupos que se establecerán en clase, los alumnos buscarán textos de prensa en los que se pueden observar clippings, blends y acronyms. Señalarán los ejemplos en los textos y escribirán un esquema en el que se describirán las principales características de cada palabra. Siempre que sea posible, los textos se presentarán en clase y se establecerá un debate en el que se comentarán las principales características de cada texto. A nuestro juicio, trabajar con textos reales en los que los alumnos puedan observar los procesos de formación de palabras en inglés es fundamental para que puedan establecer una relación entre lo estudiado en la asignatura y la vida cotidiana. Trabajar con textos de prensa les ofrece la posibilidad de observar la utilidad de los procesos morfológicos empleados por cuestiones de economía lingüística o para dotar a la lengua de viveza o expresividad. Ofrecer a los alumnos la posibilidad de comentar en clase las características de los textos trabajados permite a los alumnos expresar sus opiniones en el aula y expresar su propia interpretación de los textos basándose en argumentos lingüísticos relacionados con lo aprendido en la asignatura.

Además de entregar cada una de las tareas en el plazo establecido por la profesora, se pidió a los alumnos que enumeraran cada una de las dificultades que habían encontrado para llevarlas a cabo. Por otro lado, se les dio la posibilidad de enumerar lo que han aprendido con cada una de ellas y se les pidió que anotaran al final de cada actividad qué nota esperaban sacar y el porqué, de modo que además de convertir al alumno en responsable de su propio aprendizaje, también asume un papel activo en su evaluación. Con las actividades propuestas es evidente que cada portfolio va a ser diferente pues con las actividades se fomenta la creatividad del alumno, en palabras de Colen et al. (2006:51):

Finalmente, si entendemos que cada carpeta de aprendizaje debe reflejar el proceso de aprendizaje individual de cada alumno, y dado que éste es diferente, no existe ninguna carpeta de aprendizaje idéntica a otra. La carpeta de aprendizaje como producto final, es variada y diversa, como diversos son los alumnos y diversos somos los profesores y profesoras.

Antes de presentar las conclusiones de esta investigación, hemos de señalar que los treinta alumnos que fueron evaluados por medio del portfolio que consistía en las cuatro tareas descritas en esta sección, aprobaron la asignatura y el $60 \%$ de esos alumnos obtuvieron una nota media de notable. Por el contrario, entre los alumnos que fueron evaluados por medio del examen final encontramos un $15 \%$ de suspensos y sólo un $30 \%$ de ese grupo de alumnos obtuvieron una nota media de notable. Estos datos ponen de manifiesto que por medio del portfolio los alumnos aprenden más y mejor aunque esto lleva consigo una carga de trabajo añadido que se ve reflejada en su nota.

\section{Conclusiones}

El portfolio constituye una excelente herramienta docente para evaluar el nivel de adquisición de competencias ("saber hacer") de los alumnos, pues, tal y como se puede ver en el catálogo de actividades propuestas en el apartado anterior, se potencia que el alumno sea capaz de trabajar en grupo, seleccionar fuentes de información y tener capacidad crítica. 
Las principales ventajas que los profesores encuentran al emplear el portfolio como herramienta de evaluación son las siguientes: es una herramienta que permite articular diferentes actividades, permite a los alumnos reflexionar sobre su aprendizaje y hacer que éstos lleven a cabo un trabajo activo; además permite a los profesores evaluar de una manera muy fiable pues proporciona información al profesorado sobre diferentes momentos del proceso de enseñanza-aprendizaje y se puede observar la evolución de los alumnos. Además, el portfolio permite al profesor compartir su protagonismo y su poder con los estudiantes. Esto lleva consigo que el profesor tenga que revisar su propia metodología y tenga que hacer muy explícitos los criterios de evaluación.

Todos los alumnos que han sido evaluados con el portfolio coinciden en que han aprendido mucho y han podido ver claramente cuál es la relación entre la teoría y la práctica. Son conscientes de que han aprendido de forma progresiva y valoran las ventajas de trabajar de forma continua y no sólo unos días antes del examen. Todo esto hace que se hayan sentido parte activa del proceso de enseñanza-aprendizaje dejando a un lado el papel pasivo que normalmente lleva consigo el modo de evaluación tradicional.

El portfolio permite que el alumno sea creativo pues tiene bastante libertad para organizar su carpeta de trabajo en función de sus propios intereses y de su grado de implicación con las tareas desarrolladas. Además, el portfolio ofrece a los alumnos la posibilidad de reflexionar sobre su propio aprendizaje ya que pueden observar cuáles con sus progresos, qué dificultades tienen con algunas de las actividades propuestas, y sobre todo, les ofrece una evaluación fiable de sus aprendizajes y esfuerzos.

La principal desventaja del uso del portfolio está relacionada con el tiempo que a los alumnos les lleva hacer las actividades que forman el portfolio, hecho que hace que no siempre les sea posible tener la carpeta de aprendizaje al día. De manera similar, como docentes la principal desventaja de este modo de evaluación es el tiempo que nos lleva preparar cada una de esas actividades y evaluarlas.

En esta investigación, la evaluación no se considera un fin en sí misma sino un medio para que mejore el proceso de enseñanza-aprendizaje de modo que tanto alumnos como profesores se vean beneficiados. Por otro lado, la realización del portfolio encaja perfectamente con la filosofía del EEES pues evaluar a los alumnos de este modo implica que el eje central es el alumno y su proceso de aprendizaje.

\section{REFERENCIAS}

Brown, H.D. (2001). Teaching by Principles: An Interactive Approach to Language Pedagogy. Englewood Cliffs, N.J.: Prentice Hall Regents.

Celce Murcia, M. y E. Olshtain (2000). Discourse and Context in Language Teaching - A Guide for language Teachers. Cambridge: Cambridge University Press.

Colen, M.T., N. Giné y F. Imbernon (2006). La carpeta de aprendizaje del alumnado universitario. Barcelona: Octaedro.

Council of Europe (2001). Common European Framework of Reference for Languages: Learning, Teaching and Assessment. Cambridge: Cambridge University Press. (ver http: //www.coe.int)

Escobar, C. (2001). "La evaluación", en L. Nussbaum y M. Bernaus (eds.) Didáctica de las Lenguas Extranjeras en la Educación Secundaria Obligatoria. Madrid: Síntesis, 325-358. 
González J y R. Wagenaar (2003). Tuning Educational Structures in Europe. Informe final. Proyecto Piloto-Fase 1. Bilbao: Universidad de Deusto.

Martínez Lirola, M. (2007). "El nuevo papel del profesor universitario en el proceso de convergencia europeo y su relación con la interacción, la tutoría y el aprendizaje autónomo". Porta Linguarum. Revista Internacional de Didáctica de las Lenguas Extranjeras, 7: 3143.

Martínez Lirola, M., M. Peñalver, G. Ponce, C. Puche y J.M. Santacreu (2007) "Acciones dinamizadores en la Facultad de Filosofía y Letras de la UA". Actas de las Jornadas Nacionales de Intercambio de experiencias piloto de implantación de Metodologías ECTS tituladas "Aplicaciones prácticas de la Convergencia Europea". Badajoz: Servicio de Publicaciones y Oficina de Convergencia Europea de la UEx, 1-6.

Martínez Ruiz, M.A. y N. Sauleda Parés (2005). "Las universidades ante la necesidad dual de cambio y estabilidad", en M.J. Frau Llinares y N. Sauleda Parés (eds.) Investigar en diseño curricular. Redes de docencia en el Espacio Europeo de Educación Superior. Vol. II. Alcoy: Marfil, 5-21.

MEC (2006). La organización de las enseñanzas universitarias en España. Madrid: MEC.

Pérez Paredes, P. y F. Rubio (2005). "Testing and assessment”, en D. Madrid, N. Mclaren y A. Bueno (eds.) TEFL in Secondary Education. Granada: Universidad de Granada, 605-639.

Rico Vercher, M. y C. Rico Pérez (2004). El Portfolio Discente. Alcoy: Marfil.

Vez, J.M. (2001). Formación en Didáctica de las Lenguas Extranjeras. Rosario: Homo Sapiens. 